\title{
An inverse modeling procedure to determine particle growth and nucleation rates from measured aerosol size distributions
}

\author{
B. Verheggen ${ }^{1, *}$ and M. Mozurkewich ${ }^{1}$ \\ ${ }^{1}$ Department of Chemistry and Centre for Atmospheric Chemistry, York University, Toronto ON M3J 1P3, Canada \\ * now at: Laboratory for Atmospheric Chemistry, Paul Scherrer Institute, 5232 Villigen PSI, Switzerland
}

Received: 7 December 2005 - Published in Atmos. Chem. Phys. Discuss.: 7 March 2006

Revised: 29 May 2006 - Accepted: 25 June 2006 - Published: 17 July 2006

\begin{abstract}
Classical nucleation theory is unable to explain the ubiquity of nucleation events observed in the atmosphere. This shows a need for an empirical determination of the nucleation rate. Here we present a novel inverse modeling procedure to determine particle nucleation and growth rates based on consecutive measurements of the aerosol size distribution. The particle growth rate is determined by regression analysis of the measured change in the aerosol size distribution over time, taking into account the effects of processes such as coagulation, deposition and/or dilution. This allows the growth rate to be determined with a higher timeresolution than can be deduced from inspecting contour plots ("banana-plots"). Knowing the growth rate as a function of time enables the evaluation of the time of nucleation of measured particles of a certain size. The nucleation rate is then obtained by integrating the particle losses from time of measurement to time of nucleation. The regression analysis can also be used to determine or verify the optimum value of other parameters of interest, such as the wall loss or coagulation rate constants. As an example, the method is applied to smog chamber measurements. This program offers a powerful interpretive tool to study empirical aerosol population dynamics in general, and nucleation and growth in particular.
\end{abstract}

\section{Introduction}

From the many observations of new particle formation at different locations over the globe, it is now recognized that particle nucleation occurs widely in the troposphere (Kulmala et al., 2004). Small particles have adverse health effects (Oberdorster, 2001). When these particles grow to larger sizes they can directly affect climate by contributing to light scattering

Correspondence to: M. Mozurkewich

(mozurkew@yorku.ca) and absorption (Schwartz, 1996) and can indirectly affect climate by acting as cloud condensation nuclei and, therefore, altering cloud radiative properties and cloud lifetime (Lohmann and Feichter, 2005).

Nucleation can occur in almost any environment, subject to a favourable set of conditions. These conditions include a strong source of condensable vapour, high UV radiation intensity, low aerosol surface area, high relative humidity, low temperature, and atmospheric mixing processes. It is noteworthy that of these locations, only nucleation in the free troposphere and in the vicinity of clouds seems to agree with predictions based on classical nucleation theory (Clarke et al., 1999).

It has been suggested that the diameter of the critical cluster, the smallest particle size for which the rate of condensation is larger than the rate of evaporation, is thought to be on the order of $1 \mathrm{~nm}$ (Weber et al., 1997; Kulmala et al., 2000). This cluster of a few molecules can hardly be described as being in the liquid phase, nor is it a gas. Even the use of the term "diameter" for this agglomerate of molecules is questionable (Preining, 1998). The classical nucleation theory, however, uses bulk liquid properties to describe the critical cluster and calculate the nucleation rate. Not surprisingly, there are large discrepancies between measurements (both laboratory and atmospheric) and classical nucleation theory; the discrepancy often amounts to several orders of magnitude (Wyslouzil et al., 1991; Weber et al., 1995, 1997, 1998; Andronache et al., 1997). Different parameterizations of nucleation rates give orders of magnitude different results (Kulmala and Laaksonen, 1990). Good agreement of theoretical nucleation rates with laboratory experiments has been presented (Viisanen et al., 1997), but the sulfuric acid concentrations used were much higher than is typical for the atmosphere. Since the nucleation rate is extremely sensitive to the sulfuric acid concentration (Easter and Peters, 1994), extrapolation to atmospheric values is highly uncertain. These discrepancies illustrate a need to empirically determine the

Published by Copernicus GmbH on behalf of the European Geosciences Union. 
nucleation rate from measurements, independent of theory.

In a spatially and temporally homogeneous situation the average growth rate can be deduced from the time delay between the increase in precursor concentration and ultrafine particle number (Weber et al., 1997). If the location of the precursor source is known and constant in time, the inferred transport time to the measurement site can be used to infer the growth rate (Weber et al., 1998; O'Dowd et al., 1999), although dilution and coagulation would have influenced the size distribution during the time between nucleation and measurement. These ways of determining the growth rate are limited by the special conditions they require, and they provide an estimate of the growth rate averaged over relatively long time scales.

Usually the growth rate is estimated from the evolution of the maximum particle number in the size distributions under homogeneous conditions by fitting the trajectory of highest particle concentration in a contour-plot of diameter versus time (Mäkelä et al., 1997; Kulmala et al., 1998b, 2001). By doing this, only the maximum in the particle size distribution is used, and, as is the case for the methods discussed above, the estimated growth rate is averaged over relatively long time scales, thereby masking variations in the growth rate.

McMurry and Wilson (1982) determined the growth rate by solving the growth term in the cumulative form of the General Dynamic Equation (see Sect. 2.1). This same principle was used by Verheggen and Mozurkewich (2002), who first corrected the measured size distributions for coagulation and dilution before determining the growth rate by linear interpolation in a plot of consecutive cumulative size distributions.

Since the current commercially available measurement techniques can only detect particles larger than $3 \mathrm{~nm}$ diameter, the nucleation rate is not directly measurable. Instead, many studies have reported the appearance rate of particles above a certain threshold diameter, dictated by the minimum detectable size of the instrumentation used (Weber et al., 1995; O'Dowd et al., 1998). Often, the appearance rate is deduced from the increase in total particle concentration larger than $3 \mathrm{~nm}$ diameter (O'Dowd et al., 1998). Attempts have been made to relate this appearance rate to the actual nucleation rate by estimating the amount of particle losses since the time of nucleation due to coagulation and deposition (O'Dowd et al., 1999; Kulmala et al., 2001).

In this paper a novel method is described to accurately determine the empirical particle nucleation and growth rates. The particle growth rate is determined via a non-linear regression analysis of the General Dynamic Equation (GDE) (Friedlander, 2000) to fit the measured change of the aerosol size distribution in time. This way, the growth rate is determined using a range of size intervals rather than a total number or only the maximum of the distribution, as is implicitly done when fitting the evolution of the maximum number density in a contour-plot of consecutive size distribu- tions. Knowing the growth rate as a function of time enables an estimate of the time of formation of measured particles. By integrating the losses that have occurred between time of formation and time of measurement, the number density of nucleated particles can be determined. Knowing the concentration of nucleated particles and the time interval in which they formed gives the nucleation rate. This is different from other methods that are based on fitting the nucleation rate using an aerosol dynamics model (Lehtinen et al., 2004; Sandu et al., 2005) or on correcting the appearance rate for coagulation (O'Dowd et al., 1999; Kulmala et al., 2001; Kerminen and Kulmala, 2002).

The program, called PARGAN (particle growth and nucleation), is written using IGOR Pro software (Wavemeterics Inc.) and will be described in detail below. Its application to measurements made in the Calspan environmental chamber will be discussed. This method can serve as a powerful tool to improve our understanding of nucleation by providing data on nucleation in the atmosphere that do not depend on classical nucleation theory. These data could in turn be used to develop empirically based parameterizations to the nucleation rate, for use in simulation modeling.

\section{Theory}

\subsection{General Dynamic Equation}

The General Dynamic Equation (GDE) describes the evolution of the aerosol size distribution in time. The rate of change in cumulative particle concentration, defined as the concentration of particles larger than a certain size, is used as the quantity being fit. This greatly simplifies the growth term, and it tends to dampen the effect of noise in the data. The cumulative form of the GDE is given by

$$
\begin{gathered}
\frac{\partial N_{c}\left(r_{c}\right)}{\partial t}=\int_{r_{c}}^{r_{\infty}}\left(\frac{\partial n(r)}{\partial t}\right) d r=-\int_{r_{c}}^{r_{\infty}}\left(k_{L}(r) n(r)\right) d r+g\left(r_{c}\right) n\left(r_{c}\right)+ \\
\int_{r_{c}}^{r_{\infty}}\left(\int_{r_{0}}^{r_{c}}\left(k_{C}\left(r_{1}, r_{2}\right) n\left(r_{1}\right) n\left(r_{2}\right)\left(\frac{r}{r_{2}}\right)^{2}\right) d r_{1}\right) d r- \\
2 \int_{r_{c}}^{r_{\infty}}\left(n(r) \int_{r_{0}}^{r_{\infty}}\left(k_{C}\left(r, r_{1}\right) n\left(r_{1}\right)\right) d r_{1}\right) d r
\end{gathered}
$$

where $r_{0}$ and $r_{\infty}$ are the minimum and maximum detectable radii, respectively. The size distribution function is given by $n(r)=d N(r) / d r$, where $N(r)$ is the number of particles of radius $r$ per unit volume; $N_{c}\left(r_{c}\right)$ denotes the cumulative number concentration of particles larger than $r_{c}$. Equation (1) is obtained by integrating the regular form of the GDE. For clarity of presentation, the time indexes have been omitted here, though it should be kept in mind that the size distribution function, $n$, and the particle radius growth rate, 
$g$, are a function of both radius, $r$, and time, $t$. The first term on the right hand side of Eq. (1) describes the effect of first order losses (e.g. deposition and dilution), where $k_{L}(r)$ is the size dependent first order rate constant. The second term describes the effect of growth by condensation of low vapour pressure species. The third and fourth terms describe the effect of particles being produced by coagulation of two smaller particles (of radii $r_{1}$ and $r_{2}$, where $\left(r_{1}\right)^{3}+\left(r_{2}\right)^{3}=r^{3}$ ) and particles being lost by coagulation with another particle (of radius $r_{1}$ ), respectively. The second order rate constant for coagulation of particles of radius $r_{1}$ with those of radius $r_{2}$ is given by $k_{C}\left(r_{1}, r_{2}\right)$. These processes will be discussed in more detail below.

Direct emissions also influence the ambient size distribution, which could be represented by a zero order source term in the GDE, as it is usually independent of $n(r)$. This has been omitted from Eq. (1). Nucleation is not explicitly included in Eq. (1), since with the currently available instrumentation, the minimum detectable size will be larger than the size of a critical cluster. However, its effect on $n(r)$ is implicitly included in the condensational growth term and the boundary condition at $r_{0}$, which describe how the recently nucleated particles grow into the measured size range.

\subsection{Condensation}

The condensational growth rate, $g(r, t)$, can be written as

$g(r, t)=g_{0}(t) \gamma(r)$

where $g_{0}(t)$ is the radius growth rate in the gas kinetic limit, assuming a mass accommodation coefficient of unity. Its time dependence is due to the change in concentration of the condensing species. $\gamma(r)$ is an effective uptake coefficient (i.e. the inverse resistance; Molina et al., 1996) given by a rearrangement of the equation of Fuchs and Sutugin (1970):

$$
\frac{1}{\gamma(r)}=\frac{1}{\alpha}+\frac{3 r}{4 \lambda_{\text {vap }}}-\frac{0.47 r}{r+\lambda_{\text {vap }}}
$$

where $\alpha$ is the mass accommodation coefficient for the condensing species and $\lambda_{\text {vap }}$ is the gas phase mean free path of the condensing vapour. $\lambda_{\text {vap }}$ is only weakly dependent on the nature of the condensing species, and, for use in Eq. (3), is by definition

$\lambda_{\text {vap }} \frac{3 D_{\text {vap }}}{v_{\text {vap }}}$

where $D_{\text {vap }}$ is the gas phase diffusion coefficient and $v_{\text {vap }}$ is the mean molecular speed of the condensing vapour.

The radius growth rate can be related to the concentration of the condensing species in terms of the uptake coefficient, $\gamma(r)$, via

$g(r, t)=\frac{d r}{d t}=\frac{\gamma(r) v_{\mathrm{vap}} M_{W}\left([X]-[X]_{\mathrm{sat}}\right)}{4 \rho w N_{A}}$ where $M_{W}$ is the molecular weight of the condensing vapour, $[X]$ and $[X]_{\text {sat }}$ are its actual and saturation concentration, respectively, $\rho$ is the particle density, $N_{A}$ is Avogadro's number and $w$ is the mass fraction in the particle of the condensing species.

As illustrated by Eq. (5), the net rate of condensation is proportional to the excess concentration of the condensing species above saturation. During periods of vigorous growth, the saturation concentration can be assumed negligible compared to the actual concentration. The time dependence of the growth rate is due to the change in vapour concentration, while the size dependence is due to the size dependence of the uptake coefficient, $\gamma(r)$. This size dependence disappears for particles with radii much smaller than the mean free path, $\lambda_{\text {vap }}$, which is typically about $130 \mathrm{~nm}$ at one bar. When $[X]>>[X]_{\text {sat }}$, then the Kelvin effect does not exert a significant size dependence on the net growth rate.

Condensation is a growth process for the particle and a loss process for the gas-phase condensing species. The pseudofirst order loss rate of the vapour due to condensation (also called "condensation sink") is given by

$k_{\mathrm{cond}}(t)=\int_{r_{0}}^{r_{\infty}} \gamma(r) \pi r^{2} v n(r, t) d r$

\subsection{First order loss processes}

Any first order loss process that reduces the particle concentration (e.g. deposition, dilution) can be included in the definition of $k_{L}(r)$; thus, its definition depends on the processes that it describes. For smog chamber measurements, wall loss is the main first order loss process; this depends only on particle size, if the mixing is assumed constant in time. Two first order processes relevant for smog chamber studies are discussed here: wall loss by diffusional deposition and wall loss by gravitational settling. For small particles, wall loss by diffusion is most important, while for larger particles, loss by gravitational settling is larger.

A number of chamber studies (Crump and Seinfeld, 1981; McMurry and Rader, 1985; Bienenstock, 2000; Hoppel et al., 2001) have reported a first order rate constant, $k_{\text {diff }}(r)$, for diffusional wall loss that is proportional to the square root of the Brownian diffusion coefficient, $D_{B}(r)$. Thus, $k_{\text {diff }}(r)$ is given by

$k_{\mathrm{diff}}(r)=C_{\mathrm{diff}} \sqrt{D_{B}(r)}$

where $C_{\text {diff }}$ is a proportionality constant (in $\mathrm{cm}^{-1} \mathrm{~s}^{-1 / 2}$ ) and $D_{B}(r)$ is the Brownian diffusion coefficient of a particle of radius $r$. The value of the proportionality constant, $C_{\text {diff }}$, is dependent on the dimensions of and the amount of turbulence in the chamber, and its value can only be determined empirically. 
The first order rate constant for wall loss by gravitational settling, $k_{\text {grav }}(r)$, is given by

$k_{\text {grav }}(r)=m_{\text {part }}(r) B(r) G \frac{S}{V} F_{s}$

where $m_{\text {part }}(r)$ is the mass and $B(r)$ is the mobility of a particle of radius $r, G$ is the acceleration by gravity, $S / V$ is the surface to volume ratio of the chamber, and $F_{S}$ is the dimensionless ratio of projected horizontal surface area to total surface area in the chamber.

For atmospheric measurements, $S / V$ is the inverse of the height of the planetary boundary layer, and $F_{S}$ is unity. The effect of dilution can be included by using a suitable tracer (Verheggen and Mozurkewich, 2002).

\subsection{Coagulation}

The second order coagulation rate constants, $k_{C}\left(r_{1}, r_{2}\right)$, are calculated according to Sceats (1989). Enhancement factors due to van der Waals forces are included, using a Hamaker constant of $6.4 \times 10^{-20} \mathrm{~J}$, as determined from coagulation rates measured for $\mathrm{H}_{2} \mathrm{SO}_{4}$ ( $72 \%$ by mass) particles between 49 and $127 \mathrm{~nm}$ diameter by Chan and Mozurkewich (2001). Since these rate constants are defined so as to be consistent with chemical kinetics conventions for reactions between identical particles, there is a factor of two in the coagulation loss rather than a factor of $\frac{1}{2}$ in the production term.

When only scavenging by larger particles is considered, coagulation acts as a loss term and can be treated as pseudo first order. Then the pseudo-first order coagulation rate constant, $k_{C, I}\left(r_{1}, t\right)$, is given by

$k_{C, I}\left(r_{1}, t\right)=\int_{r_{0}}^{r_{\infty}} k_{C}\left(r_{1}, r_{2}\right) n\left(r_{2}, t\right) d r_{2}$

\section{Obtaining growth rates}

Normally, the GDE would be used to calculate the evolution of particle size distributions using input parameters such as growth rate and coagulation rate constants. Here we used the measured change in the cumulative size distribution over a finite time interval, $\Delta N_{c}\left(r_{c}\right) / \Delta t$, as an input variable, and use non-linear regression analysis to determine one or more parameters, such as the growth rate. Thus, this is a form of inverse modeling.

Specifically, in this work we focus on determining the gas kinetic growth rate, $g_{0}(t)$, by fitting $\Delta N_{c}\left(r_{c}\right) / \Delta t$ as a function of particle size. By applying this procedure to a series of consecutive time intervals, the growth rate as a function of time is obtained. Effects of other processes, such as deposition or wall loss, dilution, and coagulation can also be investigated by modifying their assumed values or by fitting some of them as part of the regression analysis.
To apply the GDE to discrete size distribution data, the differentials of Eq. (1) are approximated by finite differences, both in terms of the time interval between two measurements, and the size difference between two neighbouring size bins. The integrals are evaluated as straight summations. In the following, $r$ and $r_{c}$ stand for the radius at the logarithmic centre of a size bin, and $\Delta r$ is the size bin width. All contributions to $\Delta N_{c}\left(r_{c}\right) / \Delta t$ and to $n(r)$ are evaluated at the bin centre, and are assumed to be constant over the size bin. Each SMPS scan takes a finite amount of time. The measurement time for a scan is taken to be the time at which the SMPS detected the maximum in the number density.

The two coagulation terms have one fitting parameter; this multiplies the production and loss terms by a single value, and thus always conserves volume. Though the size dependence of coagulation rate constants is considered, it is not varied by the regression analysis. Both coagulation loss and production are included in the regression analysis. These processes are often either ignored or treated only as a loss process for particle number. However, their effect is often significant, even when the size distributions are narrow and mono-modal. Coagulation with particles smaller than the minimum detectable radius is not included in the determination of the growth rate, because their concentration is not known. This may lead to an overestimation of the condensational growth rate if coagulation with those undetected particles contributes to the overall growth of the measured particles. For the application to smog chamber data, the first order losses include wall losses by diffusion and by gravitational settling; each is proportional to a single parameter $\left(C_{\text {diff }}\right.$ and density). For the condensation term, $g_{0}(t)$ (see Eq. 2) is the default fitting parameter. Other parameters, such as $\alpha$ or $D_{\text {vap }}$, could also be fit, but this is only useful if the particle size range is wide enough for there to be a significant diffusion limitation to the uptake coefficient. We can assume that $g\left(r_{\infty}\right) n\left(r_{\infty}\right)=0$, provided that the concentration at $r_{\infty}$ is very small compared to the concentration at the distribution maximum.

In Eq. (1), the change in particle number over an infinitesimal time interval, $\partial N_{c}\left(r_{c}\right) / \partial t$, is expressed in terms of $n(r, t)$, the size distribution at time $t$. However, in the finite difference approximation, the left hand side of Eq. (1) becomes the rate of change in particle number from time $t_{1}$ to time $t_{2}$. Then $n(r, t)$ is no longer precisely defined; it has to be approximated as an average of $n\left(r, t_{1}\right)$ and $n\left(r, t_{2}\right)$. The most common way of averaging amounts to taking the average value of $n\left(r, t_{1}\right)$ and $n\left(r, t_{2}\right)$ at constant radius, $r$ (e.g. McMurry and Wilson, 1982):

$n(r, t) \approx \frac{n\left(r, t_{1}\right)+n\left(r, t_{2}\right)}{2}$

Since the number density is a function of both time and radius, Eq. (10) represents an approximation to, and not the definition of, $n(r, t)$. For example, consider a narrow distribution that grows so rapidly that the change in size between 
two successive measurements is greater than the width of the distribution. Then Eq. (10) gives a bimodal distribution with peaks near the initial and final modal sizes. But the correct average, which satisfies the GDE, is a mono-modal distribution with a peak between the initial and final modal sizes and spread over the full range of initial and final sizes.

From sensitivity studies with synthetic data, we conclude that when the radius growth over a single time interval is less than $(3 \times \sigma) \mathrm{nm}$ (equivalent to a growth rate of $54 \mathrm{~nm} \mathrm{~h}^{-1}$, for a relative geometric standard deviation of $\sigma=1.5$ and a 5 min scan time), the error in the growth rate is less than $5 \%$. Therefore the approximate average as defined by Eq. (10) is used in the regression analysis.

\subsection{Finite difference approximation of the coagulation term}

The contribution of coagulation loss to $\Delta N_{c} / \Delta t$ is determined by numerically evaluating the integrals in Eq. (1). The coagulation production term is less straightforward, because of the Jacobian factor, $\left(r / r_{2}\right)^{2}$ (Williams and Loyalka, 1991). This factor accounts for the fact that the radius of the produced particle is not the sum of the radii of the two coagulating particles. It is difficult to evaluate numerically because the discreteness of the size bins makes it impossible for all the initial and final radii to correspond to bin centres. This difficulty can be avoided by recognizing the physical basis of the Jacobian factor: It describes the way that the particles produced by coagulation are spread out over the distribution. In a numerical calculation it is more natural to simply consider all possible pairs of bins and to distribute, for each pair, the coagulation products between bins in a manner that conserves both number and mass. This distribution between bins is equivalent, for a discrete distribution, to applying the Jacobian factor.

In our procedure, we choose the sizes $r_{1}$ and $r_{2}$ of the two coagulating particles to be at bin centres, named $r_{i}$ and $r_{j}$, respectively, to distinguish them from usage in the continuous version of the GDE (Eq. 1). The produced particles of radius $r=\left(\left(r_{i}\right)^{3}+\left(r_{j}\right)^{3}\right)^{1 / 3}$ are divided over two neighbouring size bins using a procedure that is similar to those used in forward modeling exercises (Toon et al., 1988; Jacobson et al., 1994). A fraction, $f_{c}$, of these particles is assigned to the size bin with centre radius $r_{\text {high }}>r$, and a fraction $\left(1-f_{c}\right)$ is assigned to the bin with centre radius $r_{\text {low }}<r$. The centre radius of the smallest size bin to be included in the cumulative number concentration is denoted by $r_{c}$. Then, in the discrete form of Eq. (1), the rate of change of the cumulative distribution due to the coagulation production term becomes

$$
\frac{\Delta N_{c}\left(r_{c}\right)}{\Delta t}=\sum_{i=b i n_{0}}^{i=b i n_{\infty}}\left(\sum_{j=b i n_{0}}^{j=b i n_{\infty}}\left(f_{c}\left(r_{c}, i, j\right) k_{C}\left(r_{i}, r_{j}\right) n\left(r_{i}\right) n\left(r_{j}\right) \Delta r_{i} \Delta r_{j}\right)\right)
$$

where

$$
f_{c}=0 \quad \text { if } \quad r_{c}<r_{\text {low }}
$$

$$
\begin{aligned}
& f_{c}=\frac{r^{3}-r_{\text {low }}^{3}}{r_{\text {high }}^{3}-r_{\text {low }}^{3}} \text { if } \quad r_{\text {low }}<r_{c}<r_{\text {high }} \\
& f_{c}=1 \text { if } r_{c}>r_{\text {high }}
\end{aligned}
$$

Particles are not allowed to be formed outside the measured size range; this requires that the size distribution extends well beyond the size range for which concentrations are significant. This contributes to internal consistency and offers a useful quality control check, in that the total coagulation loss should equal twice the total coagulation production. A full description of the numerical details is provided by Verheggen (2004).

\subsection{Weighted fitting of the GDE to the measured data}

To decrease the effects of noise in the data, more accurate measurements carry more weight in the regression analysis than less accurate measurements. Via an iterative procedure, the sum of the weighted squared differences $\left(\chi^{2}\right)$ between fitted and measured value of $\Delta N_{c} / \Delta t$ is minimized for all measured size bins simultaneously, so the resulting value of the growth rate (and/or other fitting parameters) is the optimum value taking into account all size bins (or a specified sub-range of bins). $\chi^{2}$ is defined as:

$\chi^{2}=\sum_{r_{0}}^{r_{\infty}}\left(\frac{y_{\text {fit }}\left(r_{c}\right)-y_{\text {meas }}\left(r_{c}\right)}{\sigma_{\text {meas }}\left(r_{c}\right)}\right)^{2}$

where $y_{\mathrm{fit}}\left(r_{c}\right)$ is the fitted value, $y_{\text {meas }}\left(r_{c}\right)$ is the measured value, and $\sigma_{\text {meas }}\left(r_{c}\right)$ is the standard deviation of $\Delta N_{c}\left(r_{c}\right) / \Delta t$. PARGAN uses the non-linear least-squares procedure in Igor Pro to search for the parameter values that minimize $\chi^{2}$. Igor Pro uses an implementation of the Levenberg-Marquardt algorithm, as described by Press et al. (2002). If the values of the standard deviations are good estimates of the actual errors, then $\chi^{2}$ is of the same order of magnitude as the number of degrees of freedom; larger values of $\chi^{2}$ indicate that additional sources of error were present.

We estimate $\sigma\left(r_{c}\right)$ by:

$\sigma\left(r_{c}\right)=$

$\frac{\left[\sum_{i=\operatorname{bin}_{r_{c}}}^{i=\operatorname{bin}_{\infty}}\left\{\Delta r_{i}\left(2 n_{\min }^{2}\left(r_{i}\right)+\left(n_{\text {one }}\left(r_{i}\right) n\left(r_{i}, t_{1}\right)\right)+\left(n_{\text {one }}\left(r_{i}\right) n\left(r_{i}, t_{2}\right)\right)+\left(Q n\left(r_{i}, t_{1}\right)\right)^{2}+\left(Q n\left(r_{i}, t_{2}\right)\right)^{2}\right)\right\}\right]^{\frac{1}{2}}}{t_{2}-t_{1}}$

where $n_{\min }\left(r_{i}\right)$ is the concentration, $d N\left(r_{i}\right) / d r_{i}$, at size bin $i$ corresponding to the minimum incremental number of particle counts, $n_{\text {one }}\left(r_{i}\right)$ is the concentration corresponding to one measured count in size bin $i$, and $Q$ is an empirical, size independent, dimensionless constant, discussed in the following. Here $t_{1}$ and $t_{2}$ are the times at the beginning and end of the time interval $\Delta t$. The summation in Eq. (14) is required since this is the standard deviation of a cumulative concentration for all size bins larger than the one centred around $r_{c}$. 
The first term in Eq. (14) describes the variance due to measuring only the minimum increment of counts. Depending on how the counts are measured, this increment may be greater than unity; for the Calspan measurements, this minimum increment was 14 counts. This term only contributes significantly to $\sigma\left(r_{c}\right)$ when the number of counts in the size bin is small. It accounts for the fact that a measurement of zero counts is not infinitely accurate and prevents the weight from becoming infinity when zero counts are recorded. The factor of 2 accounts for the fact that this term, as the others, is included for the measurements at both ends of the time interval.

The next two terms describe the usual variance due to counting statistics, for each measurement time, $\sigma\left(r_{i}\right)=n\left(r_{i}\right) / \sqrt{ }$ counts. The last two terms in Eq. (14) describe the variance due to uncertainty in flow rates or other sources of error that are directly proportional to the concentration. The value of the constant, $Q$, was determined empirically. At high concentrations, variations in concentration are typically $1 \%$ to $2 \%$; this is much larger than expected from counting statistics. For the Calspan experiments, $Q$ was chosen to be 0.01 , based on obtaining the correct order of magnitude for the value of $\chi^{2}$. The error bars that we report for fitting parameters are based on the scatter around the fit.

\section{Application to smog chamber measurements: wall loss, coagulation, and growth rate}

The measurements were conducted in Calspan's $590 \mathrm{~m}^{3}$ environmental chamber during October and November 1998; Details of the chamber and its instrumentation, along with results from selected case studies on the ozonolysis of $\alpha$ pinene are given by Hoppel et al. (2001). To illustrate the use of PARGAN, we apply it to an experiment in which $\mathrm{SO}_{2}$ oxidation resulted in particle nucleation and growth.

\subsection{Chamber characteristics and experimental methods}

The Calspan chamber has a total volume of $590 \mathrm{~m}^{3}$ with a diameter and height of both $9.1 \mathrm{~m}$. This provides a surface to volume ratio $S / V$ of $0.67 \mathrm{~m}^{-1}$ and a relative projected horizontal surface area $F_{S}$ of 0.167 . It has a large mixing fan and the interior is teflon coated. A filtration system lowers measured gas phase and aerosol concentrations to below detectable levels by overnight filtration. Prior to each experiment, the chamber was filtered overnight, then sealed while background particle and gas phase concentrations were monitored for one hour. Air removed from the chamber for sampling was replaced through activated charcoal and absolute particle filters.

The aerosol size distribution from 4.4 to $404 \mathrm{~nm}$ radius was measured using a NRL DMA and MetOne 1100 CNC in scanning mode. The filtered and re-circulated sheath air of the DMA was dried and the aerosol sample was removed from the chamber through a diffusion dryer. A complete scan was measured every $288 \mathrm{~s}$. The measured size distributions were corrected for particle losses in the sample lines and for reduced CPC counting efficiency at small particle sizes prior to data analysis.

\subsection{Determination of the wall loss and coagulation rates}

The growth and nucleation rates determined by PARGAN depend on the values used for the wall loss and coagulation rate constants. Those constants can be determined by applying PARGAN to experimental data obtained under conditions under which no particle growth was occurring.

Although coagulation rate constants may be calculated theoretically, there is considerable uncertainty in the results. To account for this, we introduce a dimensionless parameter, the coagulation multiplier, $C_{\text {coag }}$, by which the theoretical coagulation rate constants are multiplied in order to agree with the measurements. PARGAN enables the determination of both $C_{\text {coag }}$ and the proportionality factor for the wall loss $\left(C_{\text {diff }}\right.$ in Eq. 7$)$ by means of regression analysis. The rate of change of total particle volume and number can also be used to determine these values; the two methods give consistent results.

Both $C_{\text {coag }}$ and $C_{\text {diff }}$ are determined from an experiment conducted on 16 November 1998. The oxidation of gas phase $\mathrm{SO}_{2}$ induced particle nucleation and growth in the absence of pre-existing aerosol. The size distribution was allowed to evolve in the dark, during which time coagulation was the dominant process occurring. The air in the chamber was then circulated through a filter to produce a substantial reduction in number concentration to around $1.3 \times 10^{4} \mathrm{~cm}^{-3}$; after this, both wall loss and coagulation were expected to be important. This last segment of the experiment was used in the analysis described in the following.

First, the rate of change in particle volume and number are used to provide an estimate of the wall loss and coagulation rates. In the absence of condensation, the decrease of particle volume is caused solely by wall losses, since coagulation does not alter the total volume. To filter out the effect of random counts in the larger size bins, only contributions from particles below $50 \mathrm{~nm}$ are included in determining the measured particle volume. This includes the entire distribution, which had number and volume maxima at 14 and $17 \mathrm{~nm}$ radius, respectively. Since these are ultrafine particles, gravitational settling can safely be ignored. Figure 1 shows the measured particle volume and number as a function of time. Fitting Eq. (7), integrated over the distribution, to the observed decrease in particle volume over the time interval from 14:30 to $15: 15$ yields $C_{\text {diff }}=3.6 \times 10^{-3} \mathrm{~cm}^{-1} \mathrm{~s}^{-1 / 2}$.

The decrease in particle number is caused by both wall loss and coagulation. By assuming that there is no condensation growth, that the wall loss is given by Eq. (7) with $C_{\text {diff }}=3.6 \times 10^{-3} \mathrm{~cm}^{-1} \mathrm{~s}^{-1 / 2}$, and that the coagulation rate constants are as calculated except for a common unknown 
multiplier $\left(C_{\text {coag }}\right)$, the GDE can be numerically integrated with respect to size. This yields an equation that depends on $C_{\text {coag }}$ and provides the change in number concentration over any given time interval. Fitting this to the observed decrease in particle concentration from 14:30 to 15:15 yields $C_{\text {coag }}=1.6$.

Using these estimates of $C_{\text {diff }}$ and $C_{c o a g}$ as inputs in PARGAN gives a best fit average growth rate of $-0.02 \pm 0.32 \mathrm{~nm} \mathrm{~h}^{-1}$, as expected since no condensation should have occurred during this segment of the experiment. Regression analysis using PARGAN can also be used to determine these parameters; however, it proved to be impossible to simultaneously fit both of these parameters and the growth rate. When the condensational growth rate was set to zero, the best fit yielded $C_{\text {diff }}=3.7 \times 10^{-3} \mathrm{~cm}^{-1} \mathrm{~s}^{-1 / 2}$ and $C_{\text {coag }}=1.5$. These values are in excellent agreement with the results from inspecting the rate of change in particle volume and number, discussed above.

To examine the effect of ignoring coagulation, we set $C_{\text {coag }}=0$. This yielded $C_{\text {diff }}=8.5 \times 10^{-3} \mathrm{~cm}^{-1} \mathrm{~s}^{-1 / 2}$, significantly larger than when coagulation was included. The same value was found when the decrease in particle number was fit by an exponential decay while ignoring coagulation. This shows that the wall loss rate can be significantly overestimated when coagulation is neglected, as is often done in chamber experiments. Note that the number concentration decay plotted in Fig. 1 appears to be first order in spite of the significant effect of coagulation.

In the subsequent data analysis the wall loss by diffusion is given by $k_{\text {diff }}=\left(3.6 \times 10^{-3} \mathrm{~cm}^{-1} \mathrm{~s}^{-1 / 2}\right) \times D_{B}^{1 / 2}$. The theoretical coagulation rate constants are multiplied by a factor of 1.5 .

The use of a single coagulation multiplier is somewhat unrealistic in that deviations from calculated coagulation rate constants may vary with particle size. Sensitivity studies indicate that the fit is improved at small particle sizes by using even larger values of $C_{\text {coag. }}$. However, the data are insufficient to draw any firm conclusion about its size dependence. Organic particles are expected to have somewhat smaller Hamaker constants, but the dependence of coagulation rate constants on the Hamaker constant is not strong for Hamaker constants larger than 1e-20 J. We have no explanation for the surprisingly large coagulation rate constants found in this analysis.

Since the wall loss and coagulation rates can only be accurately determined when the condensational growth rate is zero (or accurately known), not all experiments are equally suitable to determine $C_{\text {diff }}$ and $C_{\text {coag. }}$. However, similar values of these parameters were found from regression analysis of another experiment, suggesting that the wall loss and coagulation rates were relatively constant from experiment to experiment.

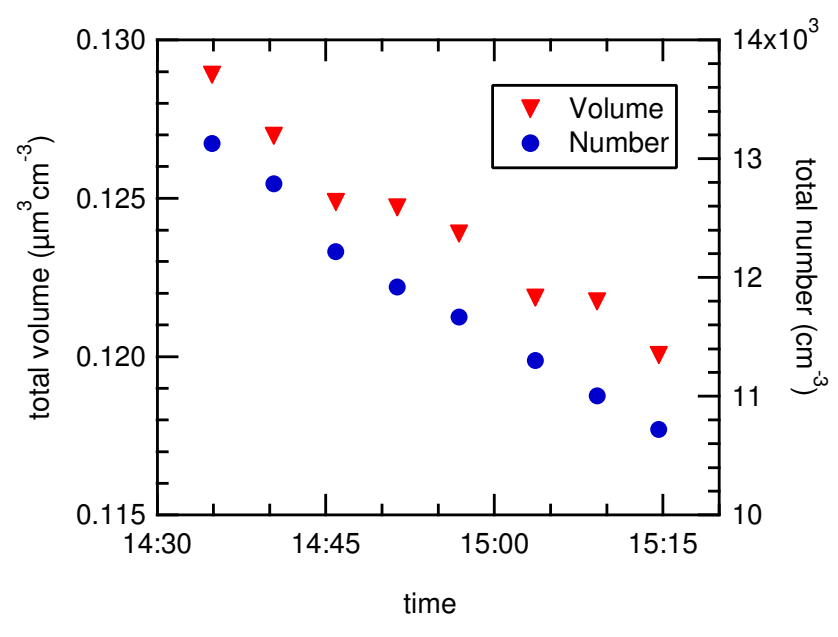

Fig. 1. Observed change in total particle volume and number during dark part of experiment, after filtering of the chamber. Due to the absence of condensation, the time dependence of the volume allows the determination of the wall loss proportionality constant, $C_{\text {diff }}$, after which the time dependence of the number concentration allows the determination of the coagulation correction factor, $C_{\text {coag }}$.

\subsection{Determination of the growth rate}

In the experiment chosen for this example, $20 \mathrm{ppb}$ of $\mathrm{CH}_{2} \mathrm{O}$ and $1.2 \mathrm{ppb}$ of $\mathrm{NO}$ were injected to generate $\mathrm{OH}$ radicals. At 10:55, $75 \mathrm{ppb}$ of $\mathrm{SO}_{2}$ was injected. Half of the chamber lights were turned on at 11:09; this started the photochemical production of $\mathrm{OH}$ and subsequent oxidation of $\mathrm{SO}_{2}$ to $\mathrm{H}_{2} \mathrm{SO}_{4}$. At 11:59, more $\mathrm{SO}_{2}$ was injected, raising the concentration from 36 to $89 \mathrm{ppb}$, and all chamber lights were turned on. This was followed by another injection of $20 \mathrm{ppb}$ of $\mathrm{SO}_{2}$ and $2 \mathrm{ppb}$ of $\mathrm{NO}$ at 12:50. The relative humidity during the experiment ranged between 75 and $82 \%$. Binary nucleation of $\mathrm{H}_{2} \mathrm{SO}_{4}$ with water vapour produced new particles that subsequently grew by condensation and coagulation. Figure 2 shows the evolution of the particle number size distribution. This nucleation and growth experiment ended when the lights were turned off at 13:37 to determine wall losses.

The mass accommodation coefficient, $\alpha$, and the diffusion coefficient of the condensing species, $D_{\text {vap }}$, both of which are included in the apparent uptake coefficient, $\gamma(r)$, were held constant at 1 and $0.1 \mathrm{~cm}^{2} \mathrm{~s}^{-1}$ respectively (Jefferson et al., 1997; Pöschl et al., 1998). The saturation concentration of the condensing species was assumed to be zero. For the experiments investigated, the magnitude of $\alpha, D_{\text {vap }}$, and saturation vapour pressure can not be realistically verified by regression analysis since the fits were insensitive to these parameters. Although the regression analysis is not sensitive to $\alpha$, the inferred concentration of condensing vapour is (see Eq. 5). A size dependence of the growth rate due to Kelvin effect was not discernible. 


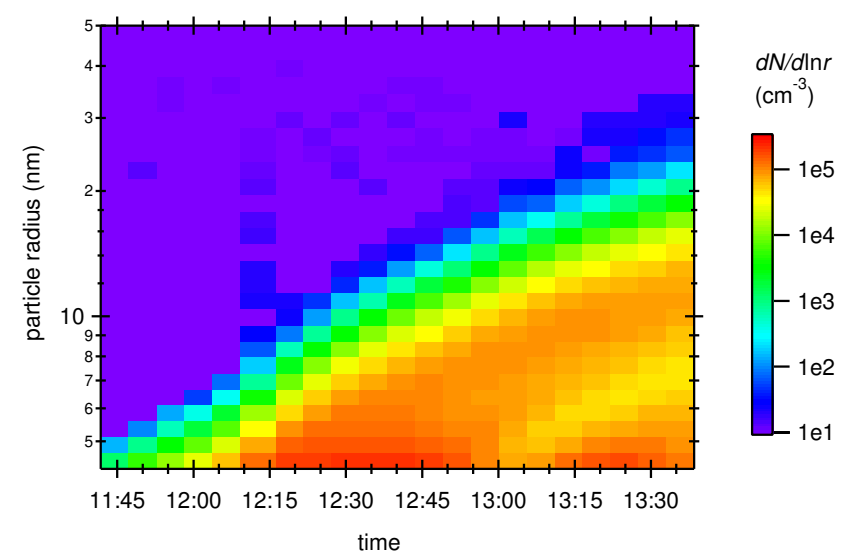

Fig. 2. Evolution of measured size distributions. Colour indicates particle number density. Half the lights were turned on at 11:09, the second half was added at 11:59. $\mathrm{SO}_{2}$ injections took place at 10:55, 11:59 and 12:50.

Results for the growth rate, obtained from regression analysis using PARGAN, are shown in Fig. 3. No growth rates could be determined prior to 11:45 since the freshly nucleated particles had not yet grown into the DMA size range. The growth rate can be related to the concentration of $\mathrm{H}_{2} \mathrm{SO}_{4}$ vapour by using Eq. (5). The values for $\rho$ and $w$ were determined by interpolating in the tables of Gmitro and Vermeulen (1964). Assuming fast equilibration at the low relative humidity $(7.5 \%)$ inside the DMA, $w$ was found to be $67 \%$ and $\rho$ was $1.57 \mathrm{~g} \mathrm{~cm}^{-3}$. Under these conditions the growth rate in the kinetic limit (assuming $\alpha=1$ ) is given by:

$g_{0}(t)=0.86 \times\left[\mathrm{H}_{2} \mathrm{SO}_{4}(t)\right]$

with $\left[\mathrm{H}_{2} \mathrm{SO}_{4}(t)\right]$ in pptv and $g_{0}(t)$ in $\mathrm{nm} \mathrm{h}^{-1}$. Figure 3 gives both the growth rate and the equivalent $\mathrm{H}_{2} \mathrm{SO}_{4}$ concentration according to Eq. (15).

As can be seen in Fig. 3, the growth rate increased immediately after the $\mathrm{SO}_{2}$ injections. The maxima in the growth rate lag behind the $\mathrm{SO}_{2}$ injections by 5 to $10 \mathrm{~min}$; this is due to the time required for the $\mathrm{H}_{2} \mathrm{SO}_{4}$ concentration to reach steady state. The time to reach steady state can be approximated by the lifetime of $\mathrm{H}_{2} \mathrm{SO}_{4}$, defined as the inverse of the pseudo-first order loss rate for condensation and wall loss. At the start of the experiment, the lifetime was around $15 \mathrm{~min}$; it decreased to approximately 4 min at 12:50 due to the increase in particle surface area. These lifetimes agree reasonably well with the observed time delay between $\mathrm{SO}_{2}$ injection and the maximum in the growth rate.

The contour plot of consecutive size distributions (Fig. 2) allows an average growth rate of $6( \pm 1) \mathrm{nm} \mathrm{h}^{-1}$ to be determined for the time interval from 12:00 to 13:30, with a slight decrease in magnitude from beginning to end. The maxima in the growth rate that are found via regression analysis (Fig. 3) are not discernible from this contour plot. We found similar results for other case studies. This suggests

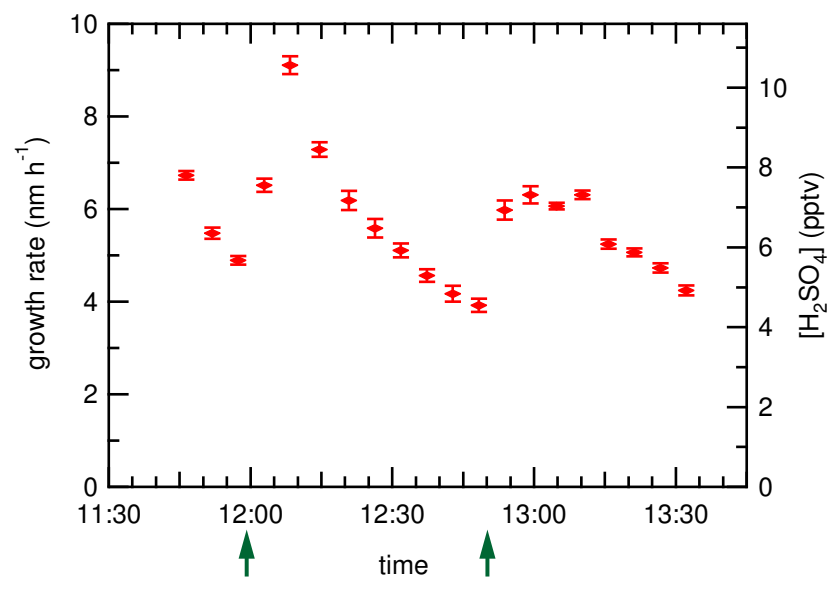

Fig. 3. The particle growth rate determined using PARGAN. The growth rate is proportional to the $\mathrm{H}_{2} \mathrm{SO}_{4}$ concentration (see Eq. 5), the corresponding values of which can be read off the right axis. Lights were turned on at 11:09; additional $\mathrm{SO}_{2}$ injections took place at 11:59 and 12:50 (indicated by arrows).

that PARGAN allows the determination of the growth rate with much better time resolution than the common method of fitting a curve through the banana-shaped contour plot of consecutive size distributions.

Although the growth rate found by regression analysis depends on to the values chosen for the coagulation and wall loss rate constants, the sensitivity is not great for the case investigated here. The growth rate changes by less than $10 \%$ if the coagulation multiplier is changed from 1.5 to 1.0 or if the wall loss rate is doubled.

Investigating other case studies from the Calspan measurements has shown that the concentration of the condensing species, as deduced from the growth rate via Eq. (5), agrees well with calculations using a simple chemical box model. This shows that an accurately known growth rate, as determined using PARGAN, can provide valuable information about the gas phase and heterogeneous chemistry of the system under investigation (Verheggen, 2004).

\section{Obtaining nucleation rates}

As a group of newly nucleated particles grow in size, their concentration changes as a result of coagulation and wall loss. We use the term "cohort" to refer to such a group of particles that are formed at approximately the same time. The determination of the nucleation rate is based on following this process backwards in time to sizes that are smaller than the minimum measured size. Starting from the measurement time, the change in cohort radius during each previous time step is determined from the growth rate. The evolution of cohort radius and number density backwards in time is evaluated for each measured size bin at each measurement time. The time when the backwards calculated radius equals 
the assumed radius, $r_{N}$, of the critical cluster $(0.5 \mathrm{~nm}$ radius, following Weber et al. (1997) and Kulmala et al. (2000)) is taken to be the time of particle formation, $t_{N}$.

The number density of the cohort is determined for each previous time step by integrating the losses that occurred in the time interval. The nucleation rate, $J$, is defined as the rate at which particles grow past the radius of the critical cluster. The number of newly nucleated particles, $n\left(r_{N}, t_{N}\right)$, is related to the nucleation rate and the growth rate via (Weber et al., 1995; Verheggen and Mozurkewich, 2002).

$J=n\left(r_{N}, t_{N}\right) g\left(r_{N}, t_{N}\right)$

In order to evaluate the time of formation and the nucleation rate, the growth rate has to be known for all time intervals between measurement time and formation time. The growth rate can only be determined from measurements when at least part of the size distribution is measured. For atmospheric measurements, these growth rates can only be used to determine the formation time and nucleation rate when the measured size distributions reflect the same air mass as that where nucleation actually took place. If those conditions are not met, but the concentration of condensing species (e.g. $\mathrm{H}_{2} \mathrm{SO}_{4}$ ) is known or can be estimated from time of formation onwards, theoretical growth rates can be calculated, and used to determine the time of formation and nucleation rate (Verheggen and Mozurkewich, 2002).

\subsection{Evaluation of number density backwards in time}

The loss terms of the GDE are applied to a cohort of particles, as they grow in size. The concentration of particles at the critical cluster size is determined by numerically integrating the pseudo-first order losses that occurred between the time of nucleation $\left(t_{N}\right.$ with radius $\left.r_{N}\right)$ and the time of measurement $\left(t_{M}\right.$ with radius $\left.r_{M}\right)$. To this end, the measured particle number density is multiplied by a correction factor for wall losses by diffusion, $D F$, and a correction factor for coagulation scavenging, $C F$ :

$n\left(r_{N}, t_{N}\right)=n\left(r_{M}, t_{M}\right) \times D F \times C F$

where

$D F=\exp \left(\int_{t_{N}}^{t_{M}} k_{\mathrm{diff}}(r, t) d t\right)=\exp \left(\int_{v_{N}}^{r_{M}} \frac{k_{\mathrm{diff}}(r, t)}{g(r, t)} d r\right)$

and

$C F=\exp \left(\int_{t_{N}}^{t_{M}} k_{C, I}(r, t) d t\right)=\exp \left(\int_{v_{N}}^{r_{M}} \frac{k_{C, I}(r, t)}{g(r, t)} d r\right)$

where $k_{\text {diff }}(r, t)$ is the first order rate constant for wall losses by diffusion (Eq. 7) and $k_{C, I}(r, t)$ is the pseudo first order rate constant for coagulation scavenging (Eq. 9). The time dependence of $k_{\text {diff }}(r, t)$ is due to the cohort radius changing

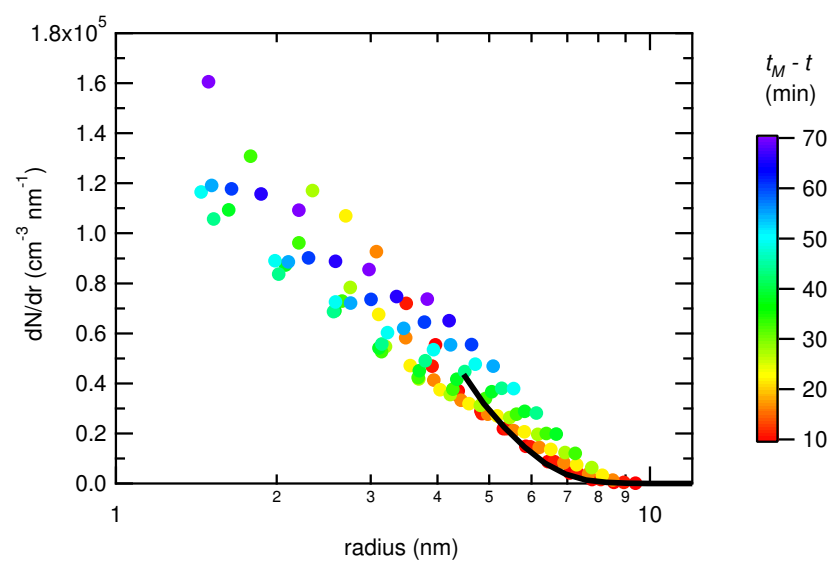

Fig. 4. Measured (solid line) and reconstructed (symbols) size distributions for 16 Nov 12:22. Reconstruction is based on size distributions, measured at later times. Colour of symbol indicates the magnitude of extrapolation, $t_{M}-t$.

in time. A different procedure is followed to obtain an additional correction factor for within-mode coagulation; this is described in Sect. 5.4.

These correction factors are equivalent to evaluating the GDE, without the growth term, as a total derivative with respect to time; the effect of growth is implicitly included via the change in cohort radius. The detailed evaluation of the correction factors will be described in subsequent sections. The correction factors can be thought of as the fractional decrease in the number density from time of nucleation to time of measurement. Note that Eqs. (17) through (19) are applied to a cohort of particles as they grow in size; thus both $r$ and $t$ are changing. The final correction factors, $D F$ and $C F$, are the product of the individual correction factors, $D F_{i}$ and $C F_{i}$, which are evaluated over the cohort radii between consecutive measurement times. This has the advantages that the growth rate can be considered constant over each time interval so that it can be taken out of the integral. Also, it enables the evaluation of correction factors for any time and/or size, an example of which is described next.

The extrapolated number concentration at a time, $t$, may be obtained by replacing $t_{N}$ with $t$ in Eqs. (17) through (19). This allows the characterization of the size distributions as they are predicted to have existed at previous time steps. The resulting "reconstructed" size distributions can be compared to the measured size distribution for the same time interval. They should all closely resemble each other if the extrapolations are accurate and internally consistent and if the measurement variability is small compared to the dynamic changes in the size distribution over time. An example of such a collection of reconstructed size distributions is given in Fig. 4. The measured distribution is matched reasonably well, especially in light of the extrapolations on which this calculation is based. The approximate treatment 
of within-mode coagulation is not included in these reconstructed size distributions.

Figure 4 also illustrates the concept of how the number of nucleated particles is determined: Similar figures depicting the reconstructed size distributions for earlier times would extend down to $0.5 \mathrm{~nm}$ radius, thus providing the number density at the time of nucleation used in Eq. (16). The reconstructed size distributions shown in Fig. 4 are valid for 12:22 and are derived from measurements made at later times. None of those measurements provided reliable data on the size distribution below $1.4 \mathrm{~nm}$. This time was chosen to provide enough overlap with the measured distribution to allow them to be compared.

\subsection{Correction for wall losses}

In the kinetic limit, the diffusion coefficient varies as $1 / r^{2}$; thus, Eq. (7) becomes $k_{\text {diff }}(r)=C / r$. Using the result of Sect. 4.2 gives $C=4.1 \times 10^{-4} \mathrm{~nm} \mathrm{~s}^{-1}$. For one time step, the correction factor for deposition, $D F_{i}$, can be solved by direct integration

$$
D F_{i}=\exp \left(\int_{r\left(t_{1}\right)}^{r\left(t_{2}\right)} \frac{k_{\mathrm{diff}}(r)}{g} d r\right)=\exp \left(\frac{C}{g} \int_{r\left(t_{1}\right)}^{r\left(t_{2}\right)} \frac{1}{r} d r\right)=\left(\frac{r\left(t_{2}\right)}{r\left(t_{1}\right)}\right)^{\frac{C}{g}}
$$

where $t_{1}<t_{2}$. Since the growth rate, $g(r, t)$, is assumed constant for the time interval from $t_{1}$ to $t_{2}$, and independent of size for the small change in cohort radius from $r\left(t_{1}\right)$ to $r\left(t_{2}\right)$, it can be taken out of the integral and is written as $g$ instead. The total correction factor for wall losses is obtained by multiplying the individual correction factors for each interval between $r_{M}$ and $r_{N}$. The first equality in Eq. (20) is generally applicable to any first order process, whereas the second and third equality are specific to wall losses of small particles in smog chamber studies.

\subsection{Correction for coagulation scavenging}

As particles grow from the critical cluster size to the measured size they undergo coagulation. We divide the coagulation events that might occur into two subsets. First, we have the coagulation of the growing cohort of particles with particles that are larger than both the cohort size and the minimum measured size. We call this "coagulation scavenging" since it is a first order loss process for the growing particles. Since we know the size distribution of the larger particles, we can explicitly calculate a pseudo-first order rate constant for the loss of particles from the growing cohort. The second subset consists of coagulation of cohort particles with particles of comparable or smaller sizes. This "within-mode coagulation" is more difficult to treat. We describe our treatment of coagulation scavenging in this section and treat within-mode coagulation in the next section.
The correction factor for coagulation scavenging, $C F_{i}$, over the time interval from $t_{1}$ (with cohort radius $r_{1}\left(t_{1}\right)$ ) to $t_{2}$ (with cohort radius $r_{1}\left(t_{1}\right)$ ) is written as

$C F_{i}=\exp \left(\frac{1}{g} \int_{r_{1}\left(t_{1}\right)}^{r_{1}\left(t_{2}\right)}\left(\int_{r_{0}^{\prime}}^{r_{\infty}}\left(k_{C}\left(r_{1}, r_{2}\right) n\left(r_{2}\right)\right) d r_{2}\right) d r_{1}\right)$

where $k_{C}\left(r_{1}, r_{2}\right)$ is the second order rate constant for coagulation and where

$r_{0}^{\prime}=r_{1}\left(t_{2}\right) \quad$ if $\quad r_{2}>r_{0}$

$r_{0}^{\prime}=r_{0} \quad$ if $\quad r_{2}<r_{0}$

Equation (21) is directly analogous to Eq. (19) where the inner integral is the pseudo-first order rate constant for scavenging of cohort particles of radius $r_{1}$ by all larger particles of radius $r_{2}$, provided that they are within the measured size range. Performing numerous evaluations of the double integral in Eq. (21) is cumbersome. The following procedure can be used to obtain an approximate expression that involves only a single integral. Since the inner integral is independent of the cohort radius the order of integration can be exchanged to obtain

$C F_{i}=\exp \left(\frac{1}{g} \int_{r_{0}^{\prime}}^{r_{\infty}}\left(n\left(r_{2}\right) \int_{r_{1}\left(t_{1}\right)}^{r_{1}\left(t_{2}\right)}\left(k_{C}\left(r_{1}, r_{2}\right)\right) d r_{1}\right) d r_{2}\right)$

In order to evaluate this integral analytically, the second order rate constant is fit to a power law of the form $k_{C}\left(r_{1}, r_{2}\right)=h\left(r_{2}\right)+c\left(r_{2}\right) \times r_{1}^{p(r 2)}$. There is a separate set of parameters $(h, c$, and $p$ ) for each discrete value of the larger radius, $r_{2}$ (corresponding to the bin centres). This power law can be analytically integrated, after which the outer integral is numerically integrated. This yields

$C F_{i}=$
$\exp \left\{\frac{1}{g} \sum_{j=\text { bin }_{0}^{\prime}}^{j=\text { bin }_{\infty}}\left[\left(h_{j}\left(r_{1}\left(t_{2}\right)-r_{1}\left(t_{1}\right)\right)+\frac{c_{j}}{p_{j}+1}\left(r_{1}\left(t_{2}\right)^{\left(p_{j}+1\right)}-r_{1}\left(t_{1}\right)^{\left(p_{j}+1\right)}\right)\right) n\left(r_{j}\right) \Delta r_{j}\right]\right\}$

The distribution function $n\left(r_{j}\right)$ is evaluated as the average between $t_{1}$ and $t_{2}$. The total correction factor for losses by coagulation scavenging is obtained by multiplying the individual correction factors for each interval between $r_{M}$ and $r_{N}$.

\subsection{Correction for within-mode coagulation}

The correction described in the previous section only accounts for coagulation with particles larger than the growing cohort. Coagulation between particles of comparable sizes both reduces the number and increases the size. We treat this "within-mode" coagulation approximately using a first order perturbation method. Since these corrections for withinmode coagulation are approximations, they bear a relatively 
large uncertainty. It is clear that if these correction factors are large (e.g. exceeding a factor of 5), the resulting values of the nucleation rate become highly uncertain, since such rapid within-mode coagulation could have erased a clear relationship between the number of nucleated and the number of measured particles.

Two different special cases, representing opposite extremes, are considered. The first limiting case assumes that growth and nucleation are in steady state, and thus constant, between the time of nucleation and the time of measurement. Consequently the size distribution function in the nucleation mode has a maximum at the critical cluster size, decreases with increasing size, and does not change with time. Nucleation may approach steady state when it is prolonged in time due to a reasonably constant super saturation of vapour.

The second limiting case assumes that there is a sharp maximum in the nucleation rate (a nucleation pulse) followed by growth of the nucleated particles. Consequently the size distribution function in the nucleation mode has a maximum at a size larger than that of the critical cluster. The pulse model assumes that the distribution consists of a cohort of identically sized particles, with a total number density of $N$. A nucleation pulse may occur due to a transient increase in vapour concentration to a value above some threshold. This is especially likely in a smog chamber, where the temperature and relative humidity are relatively constant, and more precursor gas is periodically injected.

The mathematical derivation of the limiting case of steady state nucleation is given in appendix A; the correction factor is given by

$$
\frac{n\left(r_{N}\right)}{n\left(r_{M}\right)}=b_{1}\left(r_{N}\right)\left(1+n\left(r_{M}\right) \int_{r_{N}}^{r_{M}} \frac{q(r)}{b_{1}(r)} d r\right)
$$

where

$b_{1}(r)=\exp \left(\frac{1}{g} \int_{r}^{r_{M}}\left(k_{\mathrm{diff}}\left(r^{\prime}\right)+k_{C, I}\left(r^{\prime}\right)\right) d r^{\prime}\right)$

and

$$
\begin{aligned}
q(r) & =\frac{2 b_{1}(r)}{g} \int_{r_{N}}^{r_{M}} k_{C}\left(r, r_{1}\right) b_{1}\left(r_{1}\right) d r_{1} \\
& -\frac{2}{g} \int_{r_{N}}^{r_{\text {half }}} k_{C}\left(r_{1}, r_{2}\right) b_{1}\left(r_{1}\right) b_{1}\left(r_{2}\right)\left(\frac{r}{r_{2}}\right)^{2} d r_{1}
\end{aligned}
$$

where $\left(r_{1}\right)^{3}+\left(r_{2}\right)^{3}=r^{3}$ and $g$ is the growth rate, which is assumed to be constant and independent of size for the nucleation mode particles. The second integral is symmetrical with respect to exchange of $r_{1}$ and $r_{2}$; therefore, instead of evaluating it up to $r$, it is evaluated as twice the integral up to $r_{\text {half }}=\left(r^{3} / 2\right)^{1 / 3}$. This avoids numerical instability by preventing the Jacobian factor from approaching infinity. Note that
$b_{1}\left(r_{N}\right)$ is the correction factor for pseudo-first order losses, $D F \times C F$ (Eqs. 22 and 23). The factor within brackets in Eq. (25) is the correction factor for within-mode coagulation, assuming steady state nucleation.

All integrals, except the one in Eq. (26), are determined numerically. Naturally, there is a trade-off between the accuracy of the solution and computing time. A size spacing for numerical integration of $0.5 \mathrm{~nm}$ gives satisfactory results; reducing the step size to $0.01 \mathrm{~nm}$ changes the results by less than $10 \%$.

To save computing time in the repeated calculation of $b_{1}(r)$, an approximate, analytic integration is used. The wall loss rate constant is given by Eq. (20) and the pseudo-first order rate constant for coagulation is fit to the power law $k_{C, I}(r, t)=h(t)+c(t) \times r^{p(t)}$, where the fitting parameters are a function of measurement time, $t$, and $r$ is the cohort radius. Substituting these functions into Eq. (26) yields

$$
\begin{aligned}
& b_{1}(r)=\left(\frac{r_{M}}{r}\right)^{\frac{C}{g}} \\
& \times \exp \left\{\frac{1}{g}\left(h\left(r_{M}-r\right)+\frac{c}{p+1}\left(r_{M}^{(p+1)}-r^{(p+1)}\right)\right)\right\}
\end{aligned}
$$

The growth rate and the fitting parameters are averaged over the integration interval, i.e. from the time when the cohort radius was $r$ to the time of measurement, when the cohort radius was $r_{M}$. A logarithmic average was used, since this was found to give good agreement between $b_{1}(r)$ and the first order correction factors, $C F \times D F$.

The second limiting case is that of a nucleation pulse; this is treated in detail in Appendix B. The correction factor is given by

$$
\frac{N\left(r_{N}\right)}{N\left(r_{M}\right)}=b_{1}\left(r_{N}\right)\left(1+N\left(r_{M}\right) \int_{r_{M}}^{r_{N}} p(r) d r\right)
$$

where $b_{1}(r)$ is given by Eq. (26) and

$p(r)=\frac{k_{C}(r, r) b_{1}(r)}{g^{2}}\left(\frac{1}{3} r k_{L, I}(r)-g\right)$

The quantity within brackets in Eq. (30) is the correction factor for within-mode coagulation, assuming a pulse of nucleation. Here $N\left(r_{M}\right)$ is the total number of particles in the narrow nucleation mode. As in the steady state case, $b_{1}(r)$ is calculated according to Eq. (28) and all other integrals are evaluated numerically.

\section{Determination of the nucleation rate in the smog chamber}

We now illustrate this procedure by estimating the nucleation rates corresponding to the data in Figs. 2 and 3. Nucleation rates can only be determined when the growth rate is known, that is, for the period after 11:40. Two distinct particle modes 

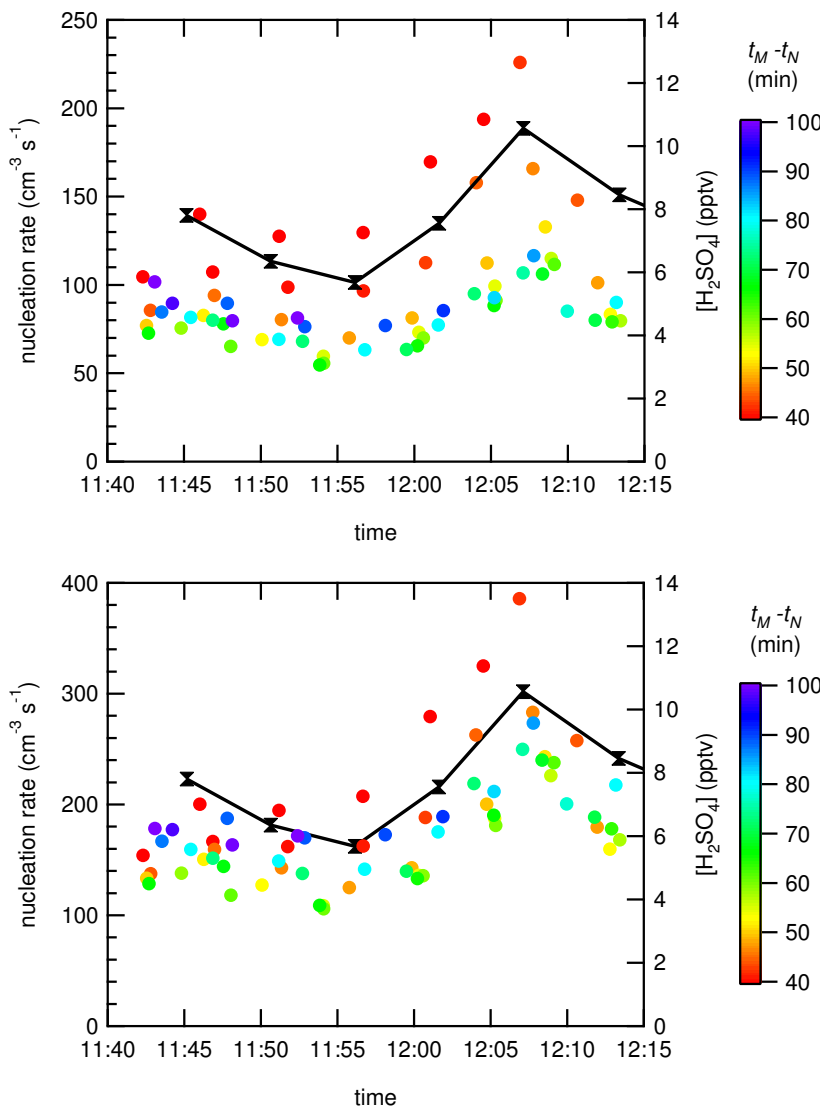

Fig. 5. Nucleation rates (coloured circles) and $\mathrm{H}_{2} \mathrm{SO}_{4}$ mixing ratios, proportional to the growth rate (black double triangles with solid line), both determined using PARGAN. Colour of symbol indicates the magnitude of extrapolation, $t_{M}-t_{N}$. (a) Excluding within-mode coagulation. (b) Including within-mode coagulation, assuming a nucleation pulse.

can be seen in Fig. 2. The first mode seems to consist of particles formed following both the first and second $\mathrm{SO}_{2}$ injections; the CPC readings show that nucleation bursts followed both injections. However, the particles formed in the first burst did not reach sizes detectable by the DMA until shortly before the second $\mathrm{SO}_{2}$ injection.

Calculated nucleation rates (only including the measured size bins which form the first mode) are shown in Figs. 5a and $b$. The former excludes the effects of within mode coagulation, whereas the latter includes these effects, assuming a pulse of nucleation. The nucleation rate can be seen to reach a modest maximum at approximately the same time as the $\mathrm{H}_{2} \mathrm{SO}_{4}$ mixing ratio (determined from the growth rate), suggesting that $\mathrm{H}_{2} \mathrm{SO}_{4}$ is responsible for both nucleation and growth. It can be seen that including within-mode coagulation almost doubles the estimated nucleation rates because it accounts for more particle losses between time of formation and time of measurement, while at the same time it reduces the scatter in the results. In contrast, using the steady-state treatment of within-mode coagulation increases the scatter, suggesting that the pulse model is more appropriate. The product of the regular correction factors, $C F \times D F$, ranges between 1.5 and 8 , where the larger values are derived from the larger measured size bins, since they required a longer extrapolation time, $t_{M}-t_{N}$, to obtain the nucleation rate. If the coagulation multiplier is set to 1 instead of to 1.5 , the nucleation rates are reduced by factors of 1.05 to 1.55 . The time evolution of the nucleation rate is not significantly affected, because of the relative insensitivity of the growth rate to changes in the coagulation (or wall loss) rate constant.

The uncertainty in the nucleation rates depends to a large degree on the amount of extrapolation necessary to obtain the correction factors $C F$ and $D F$, i.e. on $t_{M}-t_{N}$ (cf. Eqs. 18 and 19). This extrapolation time is used as a colour code in Figs. $5 \mathrm{a}$ and $\mathrm{b}$ to provide a sense of the uncertainty of the resulting nucleation rates relative to each other. The extrapolation has the effect of magnifying any uncertainty in the loss processes, i.e. in the wall loss and coagulation rate constants, as well as in the measured size distribution. The fact that all values for the nucleation rate follow approximately the same trend suggests that the input parameters have been reasonably well characterized. The uncertainty in the magnitude of the nucleation rates is estimated to be a factor of two to three, while the time dependence is estimated to be accurate within $5 \mathrm{~min}$. It is likely that the procedure results in some smearing of the time dependence of the nucleation rate.

Appearance rates for the smallest detectable size can be obtained by setting $r_{N}$ in Eqs. (18) and (19) equal to the minimum radius $(4.4 \mathrm{~nm}$ in this case). Naturally, these appearance rates are smaller than the nucleation rates. However, the time dependence of the appearance rate lags well behind that of the nucleation rate; this obscures the correlation with $\mathrm{H}_{2} \mathrm{SO}_{4}$. The ratio between the nucleation and the appearance rate (varying between 2 and 6 ) is larger than the ratio of their respective uncertainties, and therefore we regard the nucleation rate determined via this procedure to be a physically more meaningful parameter, and more representative of the "real" nucleation rate.

For the conditions at 12:00 ( $T=294 \mathrm{~K}$ and $R H=82 \%)$, the parameterization of Kulmala et al. (1998a) gives $92 \mathrm{pptv}$ as the critical $\mathrm{H}_{2} \mathrm{SO}_{4}$ mixing ratio for nucleation. This is much higher than the maximum mixing ratio of $10 \mathrm{pptv}$ deduced here. The parameterization of Kerminen and Wexler (1996) gives a critical $\mathrm{H}_{2} \mathrm{SO}_{4}$ mixing ratio of $12 \mathrm{pptv}$ for these conditions. This is only a little higher than the mixing ratios inferred from the particle growth rates and plotted in Fig. 5.

\section{Conclusions}

A comprehensive method to determine nucleation and growth rates from measured particle size distributions has been developed, named PARGAN. The growth rate is determined by non-linear regression of the GDE, taking into 
account the effect of coagulation and deposition. Using regression analysis to determine the growth rate provides better time resolution than the commonly used method of fitting the banana shaped contour plot of consecutive size distributions. Nucleation rates are determined by applying the GDE backwards in time for the measured number density in each size bin; this includes an approximate method to correct the evolution of number density for within-mode coagulation. Since PARGAN includes a full description of aerosol population dynamics, it can also be used to determine empirical rates of processes such as deposition and coagulation.

The large discrepancies between classical nucleation theory and aerosol measurements clearly show a need for an empirically based determination of nucleation rates. The method described here has the potential to be applied to a variety of situations, thereby offering the possibility of acquiring an observationally derived data set for nucleation rates in controlled laboratory environments as well as in the atmosphere. This could provide more insight in the quantitative relation between the nucleation rate and environmental factors such as precursor concentration, UV radiation intensity, particle surface area, temperature, relative humidity, and mixing. The results should be useful in developing an empirically based parameterization of the nucleation rate for use in atmospheric modeling.

The empirical nucleation rates determined via this procedure carry a large uncertainty (at least a factor of 2 to 3 ), which is partly due to the extrapolations used and partly due to the fact that the dynamics of cluster growth (e.g. the size of the critical cluster) are not well known. It should be noted that this is a huge improvement over theoretical estimates of the nucleation rate.

Application of this method to atmospheric measurements would require a careful analysis of the air mass history to evaluate the homogeneity of the air mass and the spatial scale of the nucleation event. The accuracy of the resulting nucleation and growth rates are to a large extent a reflection of the accuracy in the input data (i.e. the measured size distributions). This program offers a powerful interpretive tool to study empirical aerosol population dynamics in general, and nucleation and growth in particular.

\section{Appendix A}

\section{Correction for within-mode coagulation assuming steady state nucleation}

Here we derive a simple, analytic model for the change in concentration as particles grow from the critical cluster size to the minimum detectable size under steady state conditions. A true steady state is not needed for this to be applicable; it is only necessary that the nucleation rate remain reasonably constant over a time longer than that required for the particles to grow through this size range. At steady state, in the kinetic limit, the GDE becomes:

$$
\begin{aligned}
0 & =k_{L, I}(r) n(r)+g \frac{d n(r)}{d r}+2 n(r) \int_{r_{N}}^{r} k_{C}\left(r, r_{1}\right) n\left(r_{1}\right) d r_{1} \\
& -\int_{r_{N}}^{r} k_{C}\left(r_{1}, r_{2}\right) n\left(r_{1}\right) n\left(r_{2}\right)\left(\frac{r}{r_{2}}\right)^{2} d r_{1}
\end{aligned}
$$

where $g$ is the growth rate, which is assumed to be constant and independent of size for growth through the nucleation size range from $t_{N}$ to $t_{M}$. Coagulation scavenging by particles with radii greater than the cohort radius, $r$, is included in $k_{L, I}(r)$ as a pseudo-first order process; the coagulation terms in Eq. (A1) refer to coagulation with particles smaller than the cohort radius.

The cohort particle number density at a previous time, $n(r)$, can be estimated from its measured value at a later time, $n\left(r_{M}\right)$, based on a perturbation solution of the form

$$
n(r)=\sum_{i=1}^{i=\infty} b_{i}(r) n\left(r_{M}\right)^{i}=b_{1}(r) n\left(r_{M}\right)+b_{2}(r) n\left(r_{M}\right)^{2}+\ldots
$$

Using only the first two terms, it can be seen that

$$
\frac{n\left(r_{N}\right)}{n\left(r_{M}\right)} \approx b_{1}\left(r_{N}\right)+b_{2}\left(r_{N}\right) n\left(r_{M}\right)
$$

The right hand side of Eq. (A3) is the correction factor, by which the measured concentration of particles, $n\left(r_{M}\right)$, must be multiplied in order to obtain the concentration of nucleated particles, $n\left(r_{N}\right)$. Thus, $b_{1}\left(r_{N}\right)$ is the correction factor for (pseudo-) first order processes, i.e. wall loss and coagulation scavenging, and $b_{2}\left(r_{N}\right) n\left(r_{N}\right)$ is the correction factor for second order processes, i.e. within-mode coagulation.

Now we substitute Eq. (A2) into Eq. (A1) and collect like powers of $n\left(r_{M}\right)$. Each term in the resulting summation over $i$ must individually equal zero, because the summation must equal zero for all possible values of $n\left(r_{M}\right)$. The result is an infinite number of equations. The first of these equations is obtained by keeping only the terms proportional to $n\left(r_{M}\right)$. After dividing by $n\left(r_{M}\right)$, this "first-order" equation is

$0=k_{L, I}(r) b_{1}(r)+g \frac{d b_{1}(r)}{d r}$

Note that the coagulation terms do not contribute to this because those terms are second order and therefore involve at least $n\left(r_{M}\right)^{2}$. The solution to Eq. (A4) provides $b_{1}(r)$.

The next equation is obtained by keeping only the terms proportional to $n\left(r_{M}\right)^{2}$. After dividing by $n\left(r_{M}\right)^{2}$, this second order equation is

$$
\begin{aligned}
0 & =k_{L, I}(r) b_{2}(r)+g \frac{d b_{2}(r)}{d r}+2 b_{1}(r) \int_{r_{N}}^{r} k_{C}\left(r, r_{1}\right) b_{1}\left(r_{1}\right) d r_{1} \\
& -\int_{r_{N}}^{r} k_{C}\left(r_{1}, r_{2}\right) b_{1}\left(r_{1}\right) b_{1}\left(r_{2}\right)\left(\frac{r}{r_{2}}\right)^{2} d r_{1}
\end{aligned}
$$


The solution to this equation provides $b_{2}(r)$; this can be thought of as being the correction factor for within-mode coagulation. The key point is that to evaluate the coagulation terms in Eq. (A5) requires only the first order solution obtained from Eq. (A4).

The boundary conditions for Eqs. (A4) and (A5) are obtained by evaluating Eq. (A2) for $r=r_{M}$; this yields

$b_{1}\left(r_{M}\right)=1$

and

$b_{2}\left(r_{M}\right)=0$

Equation (A4) can be rearranged and integrated to give

$b_{1}(r)=\exp \left(\frac{1}{g} \int_{r}^{r_{M}} k_{L, I}\left(r^{\prime}\right) d r^{\prime}\right)$

Equation (A5) can be rewritten as

$0=\frac{k_{L, I}(r)}{g} b_{2}(r)+\frac{d b_{2}(r)}{d r}+q(r)$

where

$$
\begin{aligned}
q(r) & =\frac{2 b_{1}(r)}{g} \int_{r_{N}}^{r_{M}} k_{C}\left(r, r_{1}\right) b_{1}\left(r_{1}\right) d r_{1} \\
& -\frac{2}{g} \int_{r_{N}}^{r_{\text {half }}} k_{C}\left(r_{1}, r_{2}\right) b_{1}\left(r_{1}\right) b_{1}\left(r_{2}\right)\left(\frac{r}{r_{2}}\right)^{2} d r_{1}
\end{aligned}
$$

Note that $q(r)$ depends upon $r$ but not on $b_{2}(r)$. The solution to Eq. (A9) is

$$
\begin{aligned}
& b_{2}(r) \exp \left(\frac{1}{g} \int_{r_{M}}^{r} k_{L, I}\left(r^{\prime}\right) d r^{\prime}\right)= \\
& -\int_{r_{M}}^{r} q(r) \exp \left(\frac{1}{g} \int_{r_{M}}^{r} k_{L, I}\left(r^{\prime}\right) d r^{\prime}\right) d r
\end{aligned}
$$

If the integration in Eq. (A11) is taken from $r_{M}$ to $r_{N}$, we can substitute Eqs. (A7) and (A8) into the result and then change the order of integration to obtain

$$
\frac{b_{2}\left(r_{N}\right)}{b_{1}\left(r_{N}\right)}=\int_{r_{N}}^{r_{M}} \frac{q}{b_{1}(r)} d r
$$

We now substitute Eq. (A12) into Eq. (A3) to obtain the expression for the correction factor including within-mode coagulation, assuming steady state nucleation:

$\frac{n\left(r_{N}\right)}{n\left(r_{M}\right)}=b_{1}\left(r_{N}\right)\left(1+n\left(r_{M}\right) \int_{r_{N}}^{r_{M}} \frac{q(r)}{b_{1}(r)} d r\right)$

where $q(r)$ is given by Eq. (A10) and $b_{1}(r)$ is given by Eq. (A8).

\section{Appendix B}

\section{Correction for within-mode coagulation assuming pulse of nucleation}

Here we derive a simple, analytic model for the change in concentration as particles grow from the critical cluster size to the minimum detectable size following a brief pulse of nucleation. To do this, we assume that all the newly formed particles have about the same size. This should be applicable if most nucleation takes place during a time that is much shorter than that required for the particles to grow to detectable size. The rate of change of the total number concentration, $N(r)$, of newly nucleated particles is given by

$\frac{d N(r)}{d t}=-k_{L, I}(r) N(r)-k_{C}(r, r) N(r)^{2}$

where $r$ is the time dependent cohort radius. The particle radius changes due to both condensation and coagulation. Let $g$ indicate the constant condensational growth rate. To obtain the coagulational growth rate, $(d r / d t)_{\text {coag, }}$, we note that coagulation conserves volume, so that $\mathrm{Nr}^{3}$ is constant if there is no condensation taking place. Differentiating this constant with respect to time yields the growth rate when only coagulation is occurring:

$\left(\frac{d r}{d t}\right)_{\mathrm{coag}}=-\frac{r}{3 N(r)}\left(\frac{d N(r)}{d t}\right)_{\mathrm{coag}}=\frac{k_{C}(r, r) r N(r)}{3}$

The total rate of change of particle radius is given by the sum of the condensational and coagulational growth rates, thus

$$
\left(\frac{d r}{d t}\right)_{\text {total }}=g+\frac{1}{3} k_{C}(r, r) r N(r)
$$

Dividing Eq. (B1) by Eq. (B3) and rearranging yields

$$
\frac{d N(r)}{d r}\left(g+\frac{1}{3} k_{C}(r, r) r N(r)\right)+\left(k_{L, I}(r)+k_{C}(r, r) N(r)\right) N(r)=0
$$

which gives the rate of change of particle number with cohort radius and thus with time.

Now we proceed with a perturbation theory solution as in Appendix A, so that the correction factor will be given by Eq. (A3). Substituting Eq. (A2) into Eq. (B4) and collecting like powers of $N\left(r_{M}\right)$ provides the first-order equation, identical to Eq. (A4), and the second order equation

$g \frac{d b_{2}(r)}{d r}+\frac{d b_{1}(r)}{d r}\left(\frac{1}{3} k_{C}(r, r) r b_{1}(r)\right)+k_{L, I}(r) b_{2}(r)+k_{C}(r, r) b_{2}(r)^{2}=0$

After using Eq. (A4) to eliminate $d b_{1}(r) / d r$ and rearranging, Eq. (B5) becomes

$$
g \frac{d b_{2}(r)}{d r}+k_{L, I}(r) b_{2}(r)=\frac{k_{C}(r, r) b_{1}(r)}{g}\left(\frac{1}{3} r k_{L, I}(r)-g\right)
$$


with boundary conditions given by Eqs. (A6) and (A7). The solution to Eq. (B6) is

$$
\frac{d}{d r}\left(\frac{b_{2}(r)}{b_{1}(r)}\right)=\frac{k_{C}(r, r) b_{1}(r)}{g^{2}}\left(\frac{1}{3} r k_{L, I}(r)-g\right) \equiv p(r)
$$

If $r k_{L, I}(r)=3 g$, then $p(r)=0$ and the within-mode coagulation correction is zero; in this case the effect of within-mode coagulation on the rate of change of particle number (second term of Eq. B1) is exactly balanced by its effect on the rate of change of particle size (second term of Eq. B3).

Integrating Eq. (B7) from $r_{M}$ to $r_{N}$ and using the boundary condition of Eq. (A7) yields

$$
\frac{b_{2}\left(r_{N}\right)}{b_{1}\left(r_{N}\right)}=\int_{r_{M}}^{r_{N}} p(r) d r
$$

The expression for the correction factor including withinmode coagulation, assuming a pulse of nucleation, is then given by

$$
\frac{N\left(r_{N}\right)}{N\left(r_{M}\right)}=b_{1}\left(r_{N}\right)\left(1+N\left(r_{M}\right) \int_{r_{M}}^{r_{N}} p\left(r^{\prime}\right) d r^{\prime}\right)
$$

with $p(r)$ given by Eq. (B7) and $b_{1}(r)$ given by Eq. (A8).

Acknowledgements. The authors are grateful to G. Frick, P. Caffrey and W. Hoppel from the Naval Research Laboratory in Washington D.C., USA, for providing the particle size distribution measurements used in the development of the model. Funding for this work was provided by CFCAS (Canadian Foundation for Climate and Atmospheric Science) and NSERC (National Science and Engineering Research Council).

Edited by: M. Kulmala

\section{References}

Andronache, C., Chameides, W. L., Davis, D. D., Anderson, B. E., Pueschel, R. F., Bandy, A. R., Thornton, D. C., Talbot, R. W., Kasibhatla, P., and Kiang, C. S.: Gas-to-particle conversion of tropospheric sulfur as estimated from observations in the western North Pacific during PEM-West B, J. Geophys. Res., 102, 28 511-28 538, 1997.

Bienenstock, Y.: Smog chamber studies of toluene photo-oxidation by $\mathrm{OH}$ radicals, M. Sc. thesis, York University, Canada, 2000.

Chan, T. W. and Mozurkewich, M.: Measurement of the coagulation rate constant for sulfuric acid particles as a function of particle size using tandem differential mobility analysis, J. Aerosol Sci., 32, 321-339, 2001.

Clarke, A. D., Kapustin, V. N., Eisele, F. L., Weber, R. J., and McMurry, P. H.: Particle production near marine clouds: Sulfuric acid and predictions from classical binary nucleation, Geophys. Res. Lett., 26, 2425-2428, 1999.

Crump, J. G. and Seinfeld, J. H.: Turbulent deposition and gravitational sedimentation of an aerosol in a vessel of arbitrary shape, J. Aerosol Sci., 12, 405-415, 1981.
Easter, R. C. and Peters, L. K.: Binary homogeneous nucleation: Temperature and relative humidity fluctuations, non-linearity, and aspects of new particle production in the atmosphere, $\mathrm{J}$. Appl. Meteor., 33, 775-784, 1994.

Friedlander, S. K.: Smoke, dust, and haze, Oxford Unversity Press, Oxford, 2000.

Fuchs, N. A. and Sutugin, A. G.: Highly dispersed aerosols, Ann Arbor Sci., Ann Arbor, Michigan, p. 49-60, 1970.

Gmitro, J. I. and Vermeulen, T.: Vapor-liquid equilibria for aqueous sulfuric acid, Amer. Inst. Chem. Eng. J., 10, 740-746, 1964.

Hoppel, W., Fitzgerald, J., Frick, G., Caffrey, P., Pasternack, L., Hegg, D., Gao, S., Leaitch, R., Shantz, N., Cantrell, C., Albrechcinski, T., Ambrusko, J., and Sullivan, W.: Particle formation and growth from ozonolysis of alpha-pinene, J. Geophys. Res., 106, 27 603-27 618, 2001.

Jacobson, M. Z., Turco, R. P., Jensen, E. J., and Toon, O. B.: Modeling coagulation among particles of different composition and size, Atmos. Environ., 28, 1327-1338, 1994.

Jefferson, A., Eisele, F. L., Ziemann, P. J., Weber, R. J., Marti, J. J., and McMurry, P. H.: Measurements of the $\mathrm{H}_{2} \mathrm{SO}_{4}$ mass accommodation coefficient onto polydisperse aerosol, J. Geophys. Res., 102, 19021-19028, 1997.

Kerminen, V. M. and Wexler, A. S.: The occurrence of sulfuric acid-water nucleation in plumes: Urban environment, Tellus B, 48, 65-82, 1996.

Kerminen, V. M. and Kulmala, M.: Analytical formulae connecting the "real" and the "apparent" nucleation rate and the nuclei number concentration for atmospheric nucleation events, J. Aerosol Sci., 33, 609-622, 2002.

Kulmala, M. and Laaksonen, A.: Binary nucleation of the water sulfuric acid system - comparison of classical theories with different $\mathrm{H}_{2} \mathrm{SO}_{4}$ saturation vapor pressures, J. Chem. Phys., 93, 696701, 1990.

Kulmala, M., Laaksonen, A., and Pirjola, L.: Parameterizations for sulfuric acid/water nucleation rates, J. Geophys. Res., 103, 83018307, 1998a.

Kulmala, M., Toivonen, A., Makela, J. M., and Laaksonen, A.: Analysis of the growth of nucleation mode particles observed in Boreal forest, Tellus B, 50, 449-462, 1998b.

Kulmala, M., Pirjola, U., and Makela, J. M.: Stable sulphate clusters as a source of new atmospheric particles, Nature, 404, 66-69, 2000.

Kulmala, M., Dal Maso, M., Makela, J. M., Pirjola, L., Vakeva, M., Aalto, P., Miikkulainen, P., Hameri, K., and O’Dowd, C. D.: On the formation, growth and composition of nucleation mode particles, Tellus B, 53, 479-490, 2001.

Kulmala, M., Vehkamaki, H., Petajda, T., Dal Maso, M., Lauri, A., Kerminen, V. M., Birmili, W., and McMurry, P. H.: Formation and growth rates of ultrafine atmospheric particles: a review of observations, J. Aerosol Sci., 35, 143-176, 2004.

Lehtinen, K. E. J., Rannik, U., Petaja, T., Kulmala, M., and Hari, P.: Nucleation rate and vapor concentration estimations using a least squares aerosol dynamics method, J. Geophys. Res., 109, D21209, doi:10.1029/2004JD004893, 2004.

Lohmann, U. and Feichter, J.: Global indirect aerosol effects: a review, Atmos. Chem. Phys., 5, 715-737, 2005, http://www.atmos-chem-phys.net/5/715/2005/.

Mäkelä, J. M., Aalto, P., Jokinen, V., Pohja, T., Nissinen, A., Palmroth, S., Markkanen, T., Seitsonen, K., Lihavainen, H., and Kul- 
mala, M.: Observations of ultrafine aerosol particle formation and growth in boreal forest, Geophys. Res. Lett., 24, 1219-1222, 1997.

McMurry, P. H. and Wilson, J. C.: Growth laws for the formation of secondary ambient aerosols - implications for chemical conversion mechanisms, Atmos. Environ, 16, 121-134, 1982.

McMurry, P. H. and Rader, D. J.: Aerosol wall losses in electrically charged chambers, Aerosol Sci. Tech., 4, 249-268, 1985.

Molina, M. J., Molina, L. T., and Kolb, C. E.: Gas-phase and heterogeneous chemical kinetics of the troposphere and stratosphere, Ann. Rev. Phys. Chem., 47, 327-367, 1996.

O’Dowd, C., McFiggans, G., Creasey, D. J., Pirjola, L., Hoell, C., Smith, M. H., Allan, B. J., Plane, J. M. C., Heard, D. E., Lee, J. D., Pilling, M. J., and Kulmala, M.: On the photochemical production of new particles in the coastal boundary layer, Geophys. Res. Lett., 26, 1707-1710, 1999.

O'Dowd, C. D., Geever, M., and Hill, M. K.: New particle formation: Nucleation rates and spatial scales in the clean marine coastal environment, Geophys. Res. Lett., 25, 1661-1664, 1998.

Oberdorster, G.: Pulmonary effects of inhaled ultrafine particles, Int. Arch. Occup. Environ. Health, 74, 1-8, 2001.

Pöschl, U., Canagaratna, M., Jayne, J. T., Molina, L. T., Worsnop, D. R., Kolb, C. E., and Molina, M. J.: Mass accommodation coefficient of $\mathrm{H}_{2} \mathrm{SO}_{4}$ vapor on aqueous sulfuric acid surfaces and gaseous diffusion coefficient of $\mathrm{H}_{2} \mathrm{SO}_{4}$ in $\mathrm{N}_{2} / \mathrm{H}_{2} \mathrm{O}$, J. Phys. Chem. A, 102, 10 082-10 089, 1998.

Preining, O.: The physical nature of very, very small particles and its impact on their behaviour, J. Aerosol Sci., 29, 481-495, 1998.

Press, W. H., Teukolsy, S. A., Vettering, W. T., and Flannery, B. P.: Numerical recipes in $\mathrm{C}++$, Cambridge University Press, Cambridge, 2002.

Sandu, A., Liao, W., Carmichael, G. R., Henze, D. K., and Seinfeld, J. H.: Inverse modeling of aerosol dynamics using adjoints: Theoretical and numerical considerations, Aerosol Sci. Technol., 39, 677-694, 2005.

Sceats, M. G.: Brownian coagulation in aerosols - the role of longrange forces, J. Colloid Inter. Sci., 129, 105-112, 1989.
Schwartz, S. E.: The Whitehouse effect - Shortwave radiative forcing of climate by anthropogenic aerosols: An overview, J. Aerosol Sci., 27, 359-382, 1996.

Toon, O. B., Turco, R. P., Westphal, D., Malone, R., and Liu, M. S.: A multidimensional model for aerosols - description of computational analogs, J. Atmos. Sci., 45, 2123-2143, 1988.

Verheggen, B. and Mozurkewich, M.: Determination of nucleation and growth rates from observation of a $\mathrm{SO}_{2}$ induced atmospheric nucleation event, J. Geophys. Res., 107, 4123-4134, doi:10.1029/2001JD000683, 2002.

Verheggen, B.: Determination of particle nucleation and growth rates from measured aerosol size distributions, Ph.D. thesis, York University, Canada, 2004.

Viisanen, Y., Kulmala, M., and Laaksonen, A.: Experiments on gasliquid nucleation of sulfuric acid and wafer, J. Chem. Phys., 107, 920-926, 1997.

Weber, R. J., McMurry, P. H., Eisele, F. L., and Tanner, D. J.: Measurement of expected nucleation precursor species and 3-500-nm diameter particles at Mauna-Loa observatory, Hawaii, J. Atmos. Sci., 52, 2242-2257, 1995.

Weber, R. J., Marti, J. J., McMurry, P. H., Eisele, F. L., Tanner, D. J., and Jefferson, A.: Measurements of new particle formation and ultrafine particle growth rates at a clean continental site, J. Geophys. Res., 102, 4375-4385, 1997.

Weber, R. J., McMurry, P. H., Mauldin, L., Tanner, D. J., Eisele, F. L., Brechtel, F. J., Kreidenweis, S. M., Kok, G. L., Schillawski, R. D., and Baumgardner, D.: A study of new particle formation and growth involving biogenic and trace gas species measured during ACE 1, J. Geophys. Res., 103, 16 385-16396, 1998.

Williams, M. M. R. and Loyalka, S. K.: Aerosol science: theory and practice, with special applications to the nuclear industry, Elsevier Science, 116-117, 1991.

Wyslouzil, B. E., Seinfeld, J. H., Flagan, R. C., and Okuyama, K.: Binary nucleation in acid water systems 2: Sulfuric acid water and a comparison with methanesulfonic acid water, J. Chem. Phys., 94, 6842-6850, 1991. 